DOI: $10.35598 / \mathrm{mcfpga} .2019 .009$

\title{
Intelligent Lighting Control and Management System
}

\author{
Sergiy Novoselov \\ Department of Computer-Integrated Technologies, Automation \\ and Mechatronics \\ Kharkiv National University of Radio Electronics \\ Kharkiv, Ukraine \\ sergiy.novoselov@nure.ua
}

\begin{abstract}
This paper discusses the features of building an automated lighting control system. The architecture of the automated system is given. The block diagram is considered and its components are described. The principle of interaction of intelligent devices with the server is given. Presented the rationale and benefits from the introduction of such a system.
\end{abstract}

Keywords-light control, intelligent, led, network module, monitoring, server, IIoT

\section{INTRODUCTION}

At the moment, there are many organizations with no control system and electricity accounting. Installation of this automated lighting control system does not require global interference with the interior of the room. Automated lighting control systems is a complex product with a modular structure and a versatile control program for any task. Implementation of this solution helps to save energy by:

- control of level of external illumination and presence of people in the room;

- automatic maintenance of the required level of illumination in the workplace, regardless of time of day or weather conditions;

- the use of smart next lighting to increase the safety of workers at the expense of.

The peculiarity of this system is the possibility of phased and economic modernization of the obsolete lighting system through the commissioning of separate lighting devices of the structural divisions of the enterprise. Due to the connection of special control modules you can use conventional light bulbs as part of the intelligent lighting control system.

The financial costs of installing and implementing the system will be paid back in 3 to 8 years, depending on the type of organization. The enterprise will be able to save up to $60 \%$ on electricity costs.

\section{StRuCTURE AND PRINCIPLE OF THE AUTOMATED LIGHTING CONTROL SYSTEM}

The system consists of: intelligent lighting modules with built-in current and lighting sensors, with the possibility of wired or wireless control; modular light sensors for controlling light levels in the workplace; network module;

\author{
Oksana Sychova \\ Department of Computer-Integrated Technologies, Automation \\ and Mechatronics \\ Kharkiv National University of Radio Electronics \\ Kharkiv, Ukraine \\ oksana.sychova@nure.ua
}

local or cloud server; a control program for managing the operation of the system and viewing statistics.

Fig. 1 shows the architecture of the automated system.

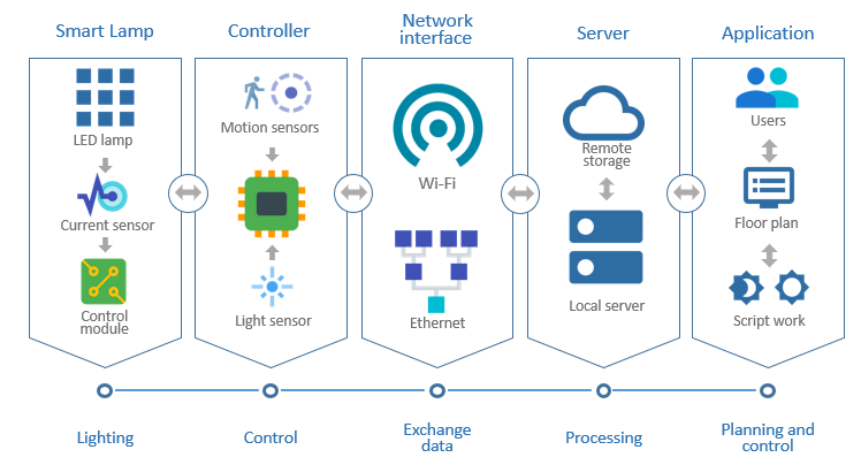

Fig. 1. Architecture of the automated system

Fig. 2 shows a schematic diagram of an automated lighting control system.

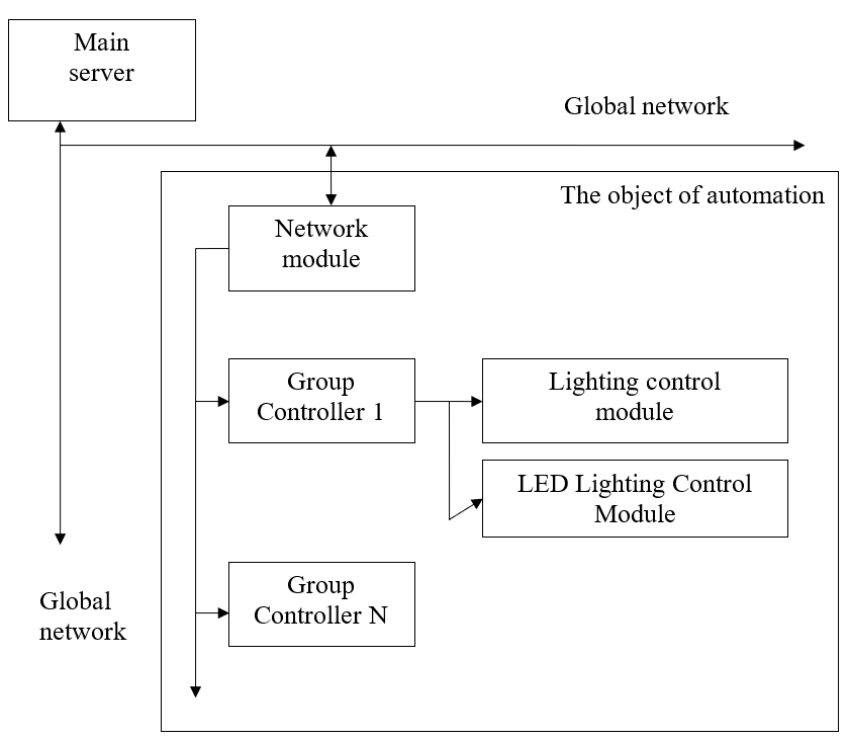

Fig. 2. The block diagram of an automated lighting control system 
The structure of the automated system includes:

- intelligent lighting control modules of different power and operating principle (LED lamps, fluorescent lamps, incandescent lamps);

- controlling controller for the management of a group of fixtures;

- network controller, which is the gateway between local lighting devices and the global network;

- emergency lighting control module and integration into automated security system.

Intelligent light control modules are separate hardware devices based on microcontrollers with built-in transceiver (with wired or wireless transmission of information).

The network module is designed to control an automated lighting control system. This module is an intermediate link between the main server and directly the lighting control modules on the object.

Fig. 3 shows the principle of the interaction of the transmitter with the server.

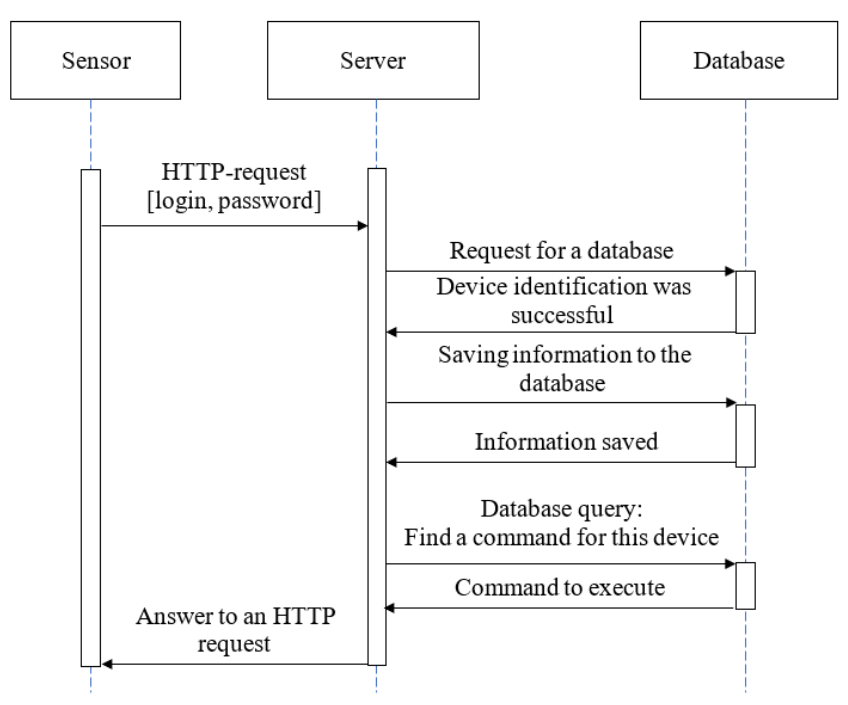

Fig. 3. The principle of the interaction of the transmitting device with the server

The network module is a gateway between the group controller that is installed on the automation object and the Internet.

Network module features:

- receiving requests from the server;

- processing received information;
- formation of packages and broadcasting of requests to the industrial network of the object of automation of lighting control;

- receive a response from the controllers of automation of lighting control;

- build response and broadcast it to the main server.

Intelligent devices send statistics to the server and expect reciprocal commands from it. In standby, the server waits for requests from controllers to read their status and save them to the database. When the device accesses the server, the latter disassembles the received message, decodes it, and records the received information to the database.

The software part of the server consists of the interface and controllers. The interface allows you to get information about the state of sensors and other devices, and manage them. The interface also allows you to change these parameters.

\section{CONCLUSIONS}

The proposed intelligent lighting control system provides the opportunity to save on the company's financial costs due to a significant reduction in electricity consumption while improving working conditions for employees.

Differences of this system from similar:

- universal solution for an object of any complexity due to;

- use of modular scalable software on the basis of micro-services;

- reducing the range of hardware to several units, which simplifies the configuration, configuration and support;

- automatic reconfiguration of the smart device network.

The use of intelligent control makes it possible to quickly detect malfunctions of lighting devices and increase the speed of response to it. Centralized control, accounting and control of the state of lighting devices, as well as control and accounting of the consumption of electricity by the lighting system, can significantly reduce the level of energy consumption.

\section{REFERENCES}

[1] O. Hersent, The Internet of Things: Key Applications and Protocols. D. Boswarthick, O. Elloumi. - Wiley, 2012. - 370 p.

[2] Gong, Y.; Han, P. Research on energy-saving scheme based on LED street lamp management-system. Appl. Mech. Mater. 2014, pp. 448453, 2850-2855.

[3] F. Behmann, Collaborative Internet of Things (C-IoT): for Future Smart Connected Life and Business. Kwok Wu K. - Wiley, 2015. $304 \mathrm{p}$. 